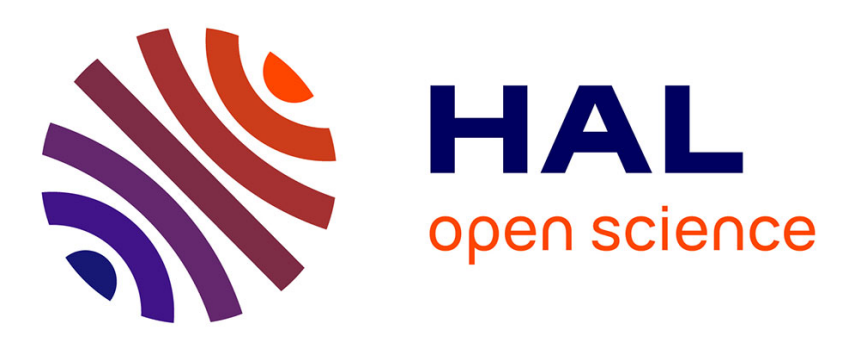

\title{
Tuning Temporal Features within the Stochastic $\pi$-Calculus
}

Loïc Paulevé, Morgan Magnin, Olivier Roux

\section{To cite this version:}

Loïc Paulevé, Morgan Magnin, Olivier Roux. Tuning Temporal Features within the Stochastic $\pi$-Calculus. IEEE Transactions on Software Engineering, 2011, 37 (6), pp.858-871. 10.1109/TSE.2010.95 . hal-00397308

\section{HAL Id: hal-00397308 \\ https://hal.science/hal-00397308}

Submitted on 20 Jun 2009

HAL is a multi-disciplinary open access archive for the deposit and dissemination of scientific research documents, whether they are published or not. The documents may come from teaching and research institutions in France or abroad, or from public or private research centers.
L'archive ouverte pluridisciplinaire HAL, est destinée au dépôt et à la diffusion de documents scientifiques de niveau recherche, publiés ou non, émanant des établissements d'enseignement et de recherche français ou étrangers, des laboratoires publics ou privés. 


\title{
Tuning Temporal Features within the Stochastic $\pi$-Calculus
}

\author{
Loïc Paulevé, Morgan Magnin, and Olivier Roux
}

\begin{abstract}
The stochastic $\pi$-calculus is a formalism that have been shown of interest for modelling systems where the stochasticity and the delay of transitions are important features, such as the biochemical reactions. Commonly, duration of transitions within stochastic $\pi$-calculus models follow an exponential random variable. Underlying dynamics of such distributed models are expressed in terms of continuous-time Markov chains and can then be efficiently simulated and model-checked. However, the exponential law comes with a huge variance making difficult the modelling of systems with accurate temporal constraints. In this report, a technique for tuning temporal features within the stochastic $\pi$-calculus is presented. This method relies on the introduction of a stochasticity absorption factor by replacing the exponential distribution by the Erlang distribution, that is the sum of exponential random variables. A construction of this stochasticity absorption factor in the classical exponentially distributed stochastic $\pi$-calculus is provided. This report also offers tools for manipulating the stochasticity absorption factor and its link with timed intervals for firing transitions. Finally, the model-checking of such designed models is tackled through the support for the stochasticity absorption factor in the stochastic $\pi$-calculus translation to the probabilistic model checker PRISM.
\end{abstract}

Index Terms-Temporal parameters, $\pi$-Calculus, Model checking, Markov processes, Stochastic processes.

\section{INTRODUCTION}

B $Y$ the introduction of temporal and stochastic aspects within models, the designer aims at reproducing real systems more closely. This complexation of models generally raises a compromise between a precise specification of temporal parameters and efficient analyses techniques.

The $\pi$-calculus [1] is a concurrent process algebra suitable for modelling independently defined entities communicating through mobile channels. This formalism is widely used for analysing, for instance, communication protocols. Amongst the different extensions of this calculus, the stochastic $\pi$-calculus [2] introduces stochastic and temporal features within $\pi$-calculus models. The stochastic $\pi$-calculus is notably used to model biological systems [3], [4], [5], [6]. Temporal and stochastic features of biochemical reactions are prominent for analysing and predicting the behaviours of such complex systems.

In the stochastic $\pi$-calculus framework, time and stochasticity are injected by making the duration of transitions to follow an exponential distribution. The parameter of this distribution is called the use rate, and informally, defines the number of times the transition can by used within a time unit. Underlying semantics of stochastic $\pi$-calculus expressions can be expressed with continuous-time Markov chains (CTMCs). By exploiting the memoryless property of the exponential law, very efficient simulation algorithms have been designed such as the Gillespie's algorithm [7], BioSpi [3] and SPiM [8]. The model checking of stochastic $\pi$-calculus has been

- IRCCyN, UMR CNRS 6597, École Centrale de Nantes, France. E-mail: \{pauleve,magnin,roux\}@irccyn.ec-nantes.fr recently proposed by [9] which offers a translation of the exponentially distributed $\pi$-Calculus to PRISM, a probabilistic symbolic model checker.

However, despite use rates bring temporal features within models, the high variance of the exponential distribution seems to prevent a precise modelling of temporal constraints. As an example, one may wonder how to model in stochastic $\pi$-calculus a transition taking place only within a given time interval, as usually done with Timed Automata [10].

In this report, we present a technique to tune temporal features within the stochastic $\pi$-calculus and we provide a method to analyse such models with PRISM. This tuning permits to model systems with both stochastic and temporal aspects in a more independent manner than in the classical stochastic $\pi$-calculus. Such a modelling accuracy is obtained by attaching to actions, in addition of the use rate, a so-called stochasticity absorption factor. The higher this factor is, the more the temporal variance is reduced around the mean imposed by the use rate.

The stochasticity absorption of transitions is obtained by replacing the exponential distribution by the Erlang distribution, which is the distribution of the sum of exponential random variables. It is worth to notice that the semantics of the stochastic $\pi$-calculus with general distributions has already been studied [11]. The originality of the presented approach is to provide a correct construction of the Erlang distributed stochastic $\pi$-calculus into the exponentially distributed stochastic $\pi$-calculus. Therefore, such models still have CTMCs semantics and can be simulated and verified with the various existing tools based on the memoryless property of the exponential distribution.

As a first result of this study, the construction of 
the stochasticity absorption factor with the exponentially distributed $\pi$-calculus allows to simulate such parametrised transitions with standard simulation algorithms.

As a second result of using this stochasticity absorption factor, a transition can be parametrised by specifying a confidence time interval of firing, referred as the firing interval. In other terms, at a given confidence coefficient, to any firing interval corresponds a unique rate and a unique stochasticity absorption factor which ensure the transition is fired within this time interval.

Finally, as a third result, we provide an adaptation of the translation of the exponentially distributed $\pi$-calculus into PRISM given by Norman et al. [12] to support the stochasticity absorption factor. In that way, model checking of Erlang distributed stochastic $\pi$ calculus can be efficiently performed thanks to PRISM.

As related works, we may cite the modelling of probabilistic timed automata as Markov decision process suitable for model checking with PRISM [13]. The proposed PRISM construction, presented in the last section of this report, follows the same idea of having dedicated transitions for incrementing a clock.

Analysis of complex dynamical systems have been widely done through model-checking algorithms designed for states graphs underlying CTMCs. For this purpose, the branching temporal logic CTL was extended as a stochastic logic called CSL which was initially proposed in [14] and furthermore extended in [15]. Later again, this approach was pursued by the work of [16]. It consisted in defining a new stochastic temporal logic (named $\mathrm{CSL}^{T A}$ ) intended at limiting the explosion of complexity in analysis. The idea of this work is to use timed automata both to express the property that has to be checked and to specify the system on which it is checked. Thus, the model-checking algorithm works on products of timed automata which prevents from computing the wholly developed reachability graph of the product. Such an avoidance of building a graph the number of states of which may makes it non tractable is an idea that we have used too in a slightly different way in our work.

This report is structured as follows. Section 2 formally presents the stochastic $\pi$-calculus. Section 3 introduces the stochasticity absorption factor through the Erlang distribution. A construction of the Erlang distributed to the exponentially distributed stochastic $\pi$-calculus is provided. Section 4 establishes the parallel between the firing interval and the rate and stochasticity absorption factor of a transition. Section 5 adapts the translation from the standard stochastic $\pi$-calculus into PRISM to support the stochasticity absorption factor. Finally, Section 6 concludes this report and discusses future works.

\section{The STOCHASTIC $\pi$-CALCULUS}

The $\pi$-calculus is a concurrent process algebra where two processes communicate using a shared channel [1]. For establishing a communication, a sender process outputs on a channel and a receiver process inputs on the same channel. The sender process may outputs names on the channel to transmit data to the receiver. A name is either a value or a channel. In that way, $\pi$-calculus allows to model the mobility of the communication channels between concurrent processes.

The stochastic extension of the $\pi$-calculus affects to each action (channel input/output or internal action) a probabilistic distribution for determining the delay until it is effective [2], [11]. Usually, the exponential distribution is preferred. The parameter of this distribution is referred as the use rate for the action, and informally, gives the number of time such an action is to be fired within a time unit.

The syntax for the stochastic $\pi$-calculus used in this report is presented by Definition 1 . It is close to the commonly used definition of the stochastic $\pi$-calculus (e.g. [8], [12]).

Definition 1 (Stochastic $\pi$-Calculus $S \pi$ ). Using $P, P_{i}, Q$ to range over terms, $A$ to range over definitions of terms, $\pi$ to range over actions and $x, y, z_{1}, \ldots, z_{n}$ to range over names:

$$
\begin{aligned}
\pi::= & \tau|a y| \bar{a} y \\
P::= & \mathbf{0}|\nu x P| P|Q|[\text { cond }] P \mid \\
& \pi \cdot P \mid A\left(z_{1}, \ldots, z_{n}\right) \\
A\left(z_{1}, \ldots, z_{n}\right)::= & \sum_{i \in I} P_{i}
\end{aligned}
$$

Where $I$ is an index set, $t$ is the identifier of the internal action and cond is a boolean expression on names.

Hereafter, P.0 is abbreviated as $P, A()$ is abbreviate ad $A$ and $z_{1}, \ldots, z_{n}$ is abbreviated as $\tilde{z}$.

The actions of a process are either internal action $(\tau)$, input of $y$ on channel $a$ (ay) or free output of $y$ on channel $a(\bar{a} y)$. After performing an action $\pi$, the process evolves as $P$, noted as $\pi . P$. The term 0 denotes the null process, $P \mid Q$ the parallel compositions of processes $P$ and $Q,[$ cond $] P$ the process $P$ enabled only if cond is satisfied (generally, cond is a conjunction of tests upon names), and $\nu x P$ is the restriction of a new name to the process $P . \sum_{i \in I} P_{i}$ is a race conditions between processes $P_{i}$ : only the first fired $P_{i}$ is considered. Each race condition has a definition of the form $A(\tilde{z})=\sum_{i \in I} P_{i}$, where $\tilde{z}$ are the parameters of the process $A$.

A name $y$ is bound to a process $P$ if there exists a term of the form $\nu y$ or ay within the expression of $P$. Otherwise, the name $y$ is free. The set of bound names in a process $P$ is noted as $b n(P)$ and the set of free names as $f n(P) \cdot n(P)=f n(P) \cup b n(P)$ is the set of names present in the term $P$.

Figure 1 depicts the operational semantics of the stochastic $\pi$-calculus. Transitions are labeled by the performed action, $\tau_{t}$ denotes an internal transition identified by $t, \dot{a}$ denotes a communication on channel $a$.

We denote by $S \pi_{e}$ the stochastic $\pi$-calculus having the duration of each action $a$ following an exponential 


\begin{tabular}{|c|c|c|c|c|}
\hline $\mathrm{PRE}_{\tau}$ & $\overline{\tau_{t} \cdot P \stackrel{\tau_{t}}{\longrightarrow} P} \quad \mathrm{PRE}_{\mathrm{IN}} \overline{\text { ay.P } \stackrel{a y}{\longrightarrow} P}$ & PRE OUT & $\overline{\bar{a} y \cdot P \stackrel{\bar{a} y}{\longrightarrow} P}$ & $\operatorname{SUM} \frac{P_{j} \stackrel{\pi}{\longrightarrow} P_{j}^{\prime}}{\left(\sum_{i \in I} P_{i}\right) \stackrel{\pi}{\longrightarrow} P_{j}^{\prime}} j \in I$ \\
\hline PAR & $\frac{P \stackrel{\pi}{\longrightarrow} P^{\prime}}{P\left|Q \stackrel{\pi}{\longrightarrow} P^{\prime}\right| Q} b n(\pi) \cap f n(Q)=\emptyset$ & & & $\operatorname{COM} \frac{P \stackrel{a y}{\longrightarrow} P^{\prime} Q \stackrel{\bar{a} z}{\longrightarrow} Q^{\prime}}{P\left|Q \stackrel{\dot{a}}{\longrightarrow} P^{\prime}\{z / y\}\right| Q^{\prime}}$ \\
\hline RES & $\frac{P \stackrel{\pi}{\longrightarrow} P^{\prime}}{\nu x P \stackrel{\pi}{\longrightarrow} P^{\prime}} x \notin n(\pi)$ & & & MATCH $\frac{P \stackrel{\pi}{\longrightarrow} P^{\prime}}{[\text { cond }] P \stackrel{\pi}{\longrightarrow} P^{\prime}}$ cond \\
\hline
\end{tabular}

Fig. 1. Operational semantics for the stochastic $\pi$-calculus.

random variable of use rate $r_{a} \in \mathbb{R}+^{*}$. The probability that an action with use rate $r$ is fired within a time $t$ is $1-\exp (-r . t)$. Its average duration is $r^{-1}$ time units with a variance of $r^{-2}$. Given $x$ actions having respectively use rates $r_{1}, \ldots, r_{x}$, the probability that the $y^{t h}$ reaction is fired is $\frac{r_{y}}{r_{1}+\cdots+r_{x}}$. We also consider actions having an infinite rate $r_{a}=\infty$, i.e. which are instantaneous. Such action are always played first. If two instantaneous actions are possible, the choice of the one to fire is nondeterministic.

\section{STOCHASTICITY ABSORPTION}

To cope with the strong bind between the average duration of an action and its probability of being fired before a given time brought by the exponential law, we introduce the stochasticity absorption factor.

Instead of having the duration of an action following one exponential random variable at rate $r$, we propose to have the duration of an action following the sum of $s a$ exponential random variables at rate r.sa. This results in an unchanged average duration but a variance divided by $s a$. In this way, $s a$ stands for the stochasticity absorption factor. The obtained probabilistic distribution is known as the Erlang distribution. Therefore, the tuning of the temporal features within the stochasticity $\pi$ calculus is achieved by attaching to each action an Erlang distribution of a fixed rate and stochasticity absorption factor. We denote by $S \pi_{E r}$ such a distributed stochastic $\pi$-calculus.

This sections starts by presenting the Erlang distribution and functions to compute some standard probabilities. The construction of $S \pi_{E r}$ into $S \pi_{e}$ is then presented, demonstrating $S \pi_{E r}$ processes can be simulated using standard algorithms based on the exponential law for firing actions. Finally, this section is illustrated by a toy example.

\subsection{The Erlang distribution}

The Erlang distribution is usually defined by two parameters: the shape $k \in \mathbb{N}^{*}$ and the rate $\lambda \in \mathbb{R}_{+}^{*}$. The Erlang distribution is then the distribution of the sum of $k$ exponential random variables at use rate $\lambda$. The Erlang distribution is a particular case of the gamma distribution where the shape parameter may be any positive real.
For the sake of consistency, we refer to the Erlang distribution as the distribution of the sum of $s a$ exponential random variables of use rate $r . s a$, where $s a$ is the stochasticity absorption factor and $r$ the use rate of the non-absorbed exponential variable (i.e. when $s a=1$ ). Equivalence between these two definitions is given by the relations $k=s a$ and $\lambda=r . s a$.

We recall the probability density function (PDF) (1) and cumulative distribution function (CDF) (2) of an Erlang distribution of rate $r$ and stochasticity absorption factor sa. PDF and CDF with different stochasticity absorption factors but a constant rate are plotted in Figure 2.

$$
\begin{aligned}
f_{r, s a}(t) & =\frac{(r . s a)^{s a} t^{s a-1} \exp (-r . s a . t)}{(s a-1) !} \\
F_{r, s a}(t) & =1-\exp (- \text { r.sa.t }) \sum_{n=0}^{s a-1} \frac{(\text { r.sa.t })^{n}}{n !} .
\end{aligned}
$$

Let be an action having its duration following an Erlang distribution of use rate $r$ and stochasticity absorption factor $s a$. The average duration of this action is $r^{-1}$ with a variance of $r^{-2} s a^{-1} . F_{r, s a}(t)$ (2) gives the probability of firing the action within a time $t$. Given $x$ actions having respectively use rates $r_{1}, \ldots, r_{x}$ and stochasticity absorption factors $s a_{1}, \ldots, s a_{x}$, the probability that the $y^{t h}$ reaction will be fired is given by (3) [11].

$$
\int_{0}^{\infty} f_{r_{y}, s a_{y}}(t) \prod_{w \neq y}\left(1-F_{r_{w}, s a_{w}}(t)\right) d t .
$$

\subsection{Stochastic $\pi$-Calculus Construction}

The paper [11] shows how to simulate stochastic $\pi$ calculus models having actions following general probabilistic distributions. However, to our knowledge, no such suitable simulator have been implemented yet. Moreover, the majority of tools around the stochastic $\pi$ calculus assume the distribution of actions being exponential [3], [8], [12]. In this section, we propose a construction of $S \pi_{E r}$ processes into $S \pi_{e}$. This construction allows the simulation of $S \pi_{E r}$ processes with standard algorithms, as previously cited.

The construction is done through a map operator $\llbracket \cdot \rrbracket_{e}$ where . stands for a $S \pi_{E r}$ term. If $P$ is a $S \pi_{E r}$ term, $\llbracket P \rrbracket_{e}$ is a $S \pi_{e}$ term showing the same behaviour than $P$ (this 

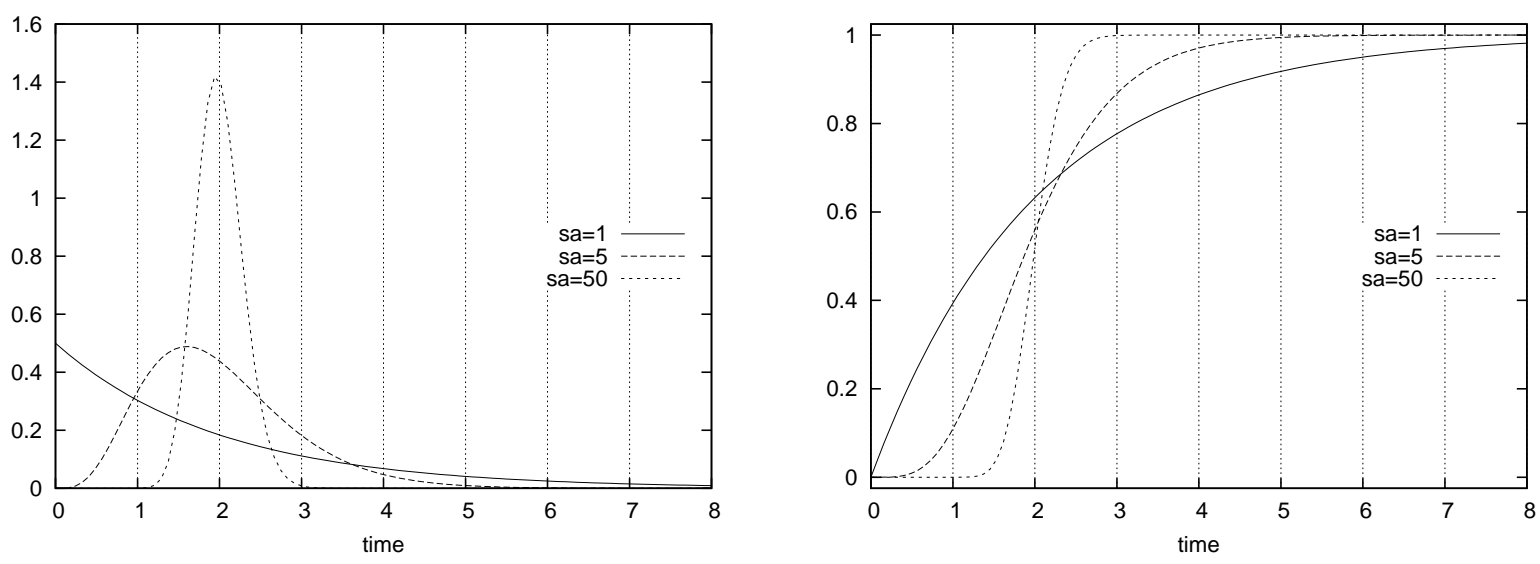

Fig. 2. Probability density function (left) and cumulative distribution function (right) of the Erlang distribution for different stochasticity absorption factors $(s a)$ when rate is $\frac{1}{2}$.

is to be more formally detailed in this section). The full mapping of $S \pi_{E r}$ terms into $S \pi_{e}$ is given by Definition 2 . It is worth to notice that this construction can be applied to any $S \pi_{E r}$ processes.

We sketch this transformation. Any action of rate $r$ has to be replaced by $s a$ actions of rate r.sa. Beforehand, to each couple of parameters $r_{a}, s a_{a}$ of the $S \pi_{E r}$ process, a parameter $r_{a}^{\prime}=r_{a} . s_{a}$ is set for the resulting $S \pi_{e}$ process. Then, the main idea is to count the number of times an actions has been effective: until this number is equal to the stochasticity absorption factor, the current process is restarted. Let $A(\tilde{z})$ be a race condition, i.e. $A(\tilde{z}):=\sum_{i \in I} P_{i}$. By definition, each term of the sum starts by an action (possibly prefixed by restriction or enabling conditions). Basically, for each of these actions, indexed by $i$, a counter $c_{i}$ is attached. This counter is given as an argument for $A$. Each time the $i^{\text {th }}$ action is fired, the counter $c_{i}$ is incremented by one, and until it reaches the given stochasticity absorption factor, the process $A$ is restarted. This principle is straightforwardly applied to the internal actions (10). Handling channel input and output is more tricky. Consider the following $S \pi_{E r}$ process $A_{1}$ :

$$
A_{1}::=\bar{a} . \mathbf{0} \quad A_{2}::=a .0 \quad A::=A_{1}\left|A_{2}\right| A_{2} .
$$

When counting the number of firing of the channel output $\bar{a}$, there must be a differentiation between responding instances of $A_{2}$. To achieve this differentiation, the $S \pi_{e}$ process $A_{1}$ initiates a private communications with the responding process by sharing a new name $a^{\prime}$. After $s a_{a}$ enabling of the private communication $a^{\prime}$, $A_{1}$ outputs on another private channel $a^{\prime \prime}$ to inform its correspondent that the correct number of communication has been done. Technically, this behaviour is achieved by storing into $c_{i}$ the set of current active private communications and the counter of firing for each (11). For the channel input action, no counting is necessary: the process first inputs the two private channels $a^{\prime}$ and $a^{\prime \prime}$ and input on them by adding race conditions. An input on $a^{\prime}$ means the stochasticity absorption is not complete, so the current process has to be restarted. An input on $a^{\prime \prime}$ means the communication is terminated, the next process is called. As for the channel output, the set of pair of private channels has to be stored in $c_{i}$ (12).

Definition $2\left(\llbracket \cdot \rrbracket_{e}\right)$. Let $\llbracket \cdot \rrbracket_{e}$ be the map from $S \pi_{E r}$ processes and definitions into $S \pi_{e}$ processes and definitions given by rules below.

Notations: $\tilde{c}$ stands for $c_{1}, \ldots, c_{n} . c_{i}$ is either a positive integer (internal action), a set of triples $\left\langle c_{i n}^{s a}, c_{i n}^{a^{\prime}}, c_{i n}^{a^{\prime \prime}}\right\rangle$ (output) $-n$ is the index of the element in the set, $c_{i n}^{s a}$ a positive integer, and $c_{i n}^{a^{\prime}}, c_{i n}^{a^{\prime \prime}}$ a couple of channels - or a set of couples of channels $\left\langle c_{i n}^{a^{\prime}}, c_{i n}^{a^{\prime \prime}}\right\rangle$ (input). $\tilde{c}\left\{c_{i}+=1\right\}$ stands for $\tilde{c}$ having $c_{i}$ incremented by one ( $c_{i}$ has to be a positive integer). \# $c_{i}$ is the size of the set $c_{i} . \bar{a}\left\langle a^{\prime}, a^{\prime \prime}\right\rangle$ (resp. $a\left\langle a^{\prime}, a^{\prime \prime}\right\rangle$ ) stands for the outputting (resp. inputting) of the couple of names $a^{\prime}, a^{\prime \prime}$.

$$
\begin{aligned}
\llbracket \mathbf{0} \rrbracket_{e} & =\mathbf{0} \\
\llbracket \nu x P \rrbracket_{e} & =\nu x \llbracket P \rrbracket_{e} \\
\llbracket P \mid Q \rrbracket_{e} & =\llbracket P \rrbracket_{e} \mid \llbracket Q \rrbracket_{e} \\
\llbracket[\text { cond }] P \rrbracket_{e} & =[\text { cond }] \llbracket P \rrbracket_{e}
\end{aligned}
$$

Process definition:

$$
\llbracket A(\tilde{z})::=\sum_{i \in I} P_{i} \rrbracket_{e}=A(\tilde{z}, \tilde{c})::=\sum_{i \in I} \llbracket P_{i} \rrbracket_{e}
$$

with $\tilde{c} \cap b n\left(\sum_{i \in I} \llbracket P_{i} \rrbracket_{e}\right)=\emptyset$.

Internal action:

$$
\begin{aligned}
\llbracket \tau_{t} \cdot P \rrbracket_{e} & =\left[c_{i}<s a_{t}\right] \tau_{t} \cdot A\left(\tilde{z}, \tilde{c}\left\{c_{i}+=1\right\}\right) \\
& +\left[c_{i}=s a_{t}\right] \tau_{t} \cdot \llbracket P \rrbracket_{e}
\end{aligned}
$$

Channel output:

$$
\begin{gathered}
\llbracket \bar{a} y \cdot P \rrbracket_{e}= \\
\nu a^{\prime} \nu a^{\prime \prime} \bar{a}\left\langle a^{\prime}, a^{\prime \prime}\right\rangle \cdot A\left(\tilde{z}, \tilde{c}^{\prime}\right) \\
+\sum_{n=0}^{\# c_{i}}\left[c_{i n}^{s a}<s a_{a}\right] \overline{c_{i n}^{a^{\prime}}} \cdot A\left(\tilde{z}, \tilde{c}\left\{c_{i n}^{s a}+=1\right\}\right)+ \\
{\left[c_{i n}^{s a}=s a_{a}\right] \overline{c_{i n}^{a^{\prime \prime}} y \cdot \llbracket P \rrbracket_{e}}}
\end{gathered}
$$


with $r_{a^{\prime}}^{\prime}=r_{a}^{\prime}$ and $r_{a^{\prime \prime}}^{\prime}=\infty,\left\{a^{\prime}, a^{\prime \prime}\right\} \cap(f n(A) \cup f n(P))=\emptyset$, and $\tilde{c}^{\prime}$ is $\tilde{c}$ where $c_{i}$ has a new element $\left\langle c^{\prime s a}, c_{i n}^{\prime a^{\prime}}, c_{i n}^{\prime^{\prime \prime}}\right\rangle=$ $\left\langle 1, a^{\prime}, a^{\prime \prime}\right\rangle, n=\# c_{i}+1$.

Channel input:

$$
\begin{aligned}
& \llbracket a y . P \rrbracket_{e}=a\left\langle a^{\prime}, a^{\prime \prime}\right\rangle \cdot A\left(\tilde{z}, \tilde{c}^{\prime}\right) \\
& \quad+\sum_{n=0}^{\# c_{j}} c_{j n}^{a^{\prime}} \cdot A(\tilde{z}, \tilde{c})+c_{j n}^{a^{\prime \prime}} y \cdot \llbracket P \rrbracket_{e}
\end{aligned}
$$

with $\left\{a^{\prime}, a^{\prime \prime}\right\} \cap(f n(A) \cup f n(P))=\emptyset$, and $\tilde{c}^{\prime}$ is $\tilde{c}$ where $c_{i}$ has a new element $\left\langle c^{\prime^{\prime}}{ }_{i n}^{\prime}, c^{\prime a^{\prime \prime}}\right\rangle=\left\langle a^{\prime}, a^{\prime \prime}\right\rangle, n=\# c_{i}+1$.

Process call:

$$
\begin{gathered}
\llbracket A(\tilde{z}) \rrbracket_{e}=A(\tilde{z}, \tilde{c}) \\
\text { with } \forall i \in I, c_{i}= \begin{cases}1 & \text { if the first action of } P_{i} \\
\emptyset & \text { is an internal action, } \\
\emptyset & \text { else. }\end{cases}
\end{gathered}
$$

\subsection{Correctness of the construction}

To ensure that this construction is correct several points have to be proved. First, the qualitative behaviour has to be preserved: there must be an equivalence between transitions from any $S \pi_{E r}$ process $A(\tilde{z})$ to a process $B(\tilde{w})$ by the action $\pi$ (noted as $A(\tilde{z}) \stackrel{\pi}{\rightarrow}_{E} B(\tilde{w})$ ) and a series of transitions starting from the $S \pi_{e}$ process $A_{e}\left(\tilde{z}, c_{\tilde{A} 0}\right)$ to the process $B_{e}\left(\tilde{w}, c_{B} 0\right)$ (denoted as $A_{e}\left(\tilde{z}, c_{\tilde{A} 0}\right) \rightarrow_{e}$ $\left.\cdots B_{e}\left(\tilde{w}, c_{B} 0\right)\right)$, where $A_{e}$ (resp. $\left.B_{e}\right)$ is the constructed $S \pi_{e}$ process from the $S \pi_{E r}$ process $A$ (resp. $B$ ) using $\llbracket . \rrbracket_{e}$ construction and $c_{\tilde{A} 0}$ and $c_{\tilde{B} 0}$ are computed by following (13). Finally, it must be ensured a transition in $S \pi_{E r}$ at rate $r$ and stochasticity absorption factor $s a$ results in $s a$ transitions at rate r.sa in $S \pi_{e}$.

Figure 3 identifies the transition rules for a $S \pi_{e}$ process resulting from the construction defined in Definition 2.

At first, the inclusion of behaviours of $S \pi_{E r}$ process in the behaviours of the constructed $S \pi_{e}$ process is proven. Let $A(\tilde{z}) \stackrel{\pi}{\rightarrow}_{E} B(\tilde{w})$ be a transition, where $\pi$ is either an internal action $t\left(\pi=\tau_{t}\right)$ or a communication on channel $a(\pi=\dot{a})$. The index of the action $\pi$ in the sum defined by $A$ is denoted by $i$.

\section{Internal action $\left(\pi=\tau_{t}\right)$}

At the initial call of $A_{e}(\tilde{z}, \tilde{c}), c_{i}=1$ (Equation (13)). As long as $c_{i}<s a_{t}$, only the rule $\mathrm{TAU}_{\text {wait }}$ can be applied. After each of these transitions, $c_{i}$ is incremented by 1 . When $c_{i}$ reaches $s a_{t}, \mathrm{TAU}_{\text {eff }}$ is the only rule applicable, resulting in a transition towards $B_{e}(\tilde{w}, \tilde{d})$.

Thus, for each $A(\tilde{z}) \stackrel{\tau_{t}}{\longrightarrow} E B(\tilde{w})$, there exists a chain:

$$
A_{e}(\tilde{z}, \tilde{c}) \stackrel{\tau_{t}}{\longrightarrow}{ }_{e} \cdots A_{e}\left(\tilde{z}, \tilde{c_{k}}\right) \stackrel{\tau_{t}}{\longrightarrow} B_{e}(\tilde{w}, \tilde{d})
$$

where $\tilde{c_{k}}$ is $\tilde{c}$ having $c_{i}=k$. From the conditions for applying $\mathrm{TAU}_{\mathrm{eff}}$, it is obvious that $k=s a_{t}$. The duration of the chain follows an Erlang distribution of rate $r_{t}^{\prime}$ and stochasticity absorption $s a_{t}$.

\section{Communication $(\pi=\dot{a})$}

We look for an equivalence of the COM transition $A(\tilde{z})\left|C\left(\tilde{z}^{\prime}\right) \stackrel{\dot{a}}{\rightarrow}_{E} B(\tilde{w})\right| D\left(\tilde{w}^{\prime}\right)$ in the $S \pi_{e}$ translated processes $A_{e}, C_{e}, B_{e}, D_{e}$. We assume $A(\tilde{z}) \stackrel{a y}{\longrightarrow} B(\tilde{w})$ and $C\left(\tilde{z}^{\prime}\right) \stackrel{\bar{a} y}{\longrightarrow} D\left(\tilde{w}^{\prime}\right)$.

Initially, only rules $\mathrm{IN}_{\text {init }}$ and $\mathrm{OUT}_{\text {init }}$ can be applied:

$$
A_{e}(\tilde{z}, \tilde{c})\left|C_{e}\left(\tilde{z}^{\prime}, \tilde{c}^{\prime}\right) \stackrel{\dot{a}}{\rightarrow} A_{e} A_{e}\left(\tilde{z}, \tilde{c}_{1}\right)\right| C_{e}\left(\tilde{z}^{\prime}, \tilde{c}_{1}^{\prime}\right)
$$

where $\tilde{c}_{1}$ and $\tilde{c}_{1}^{\prime}$ are computed according to (12) and (11). During this communication, $B_{e}$ sends to $A_{e}$ two fresh names $a^{\prime}$ and $a^{\prime \prime}$. These names are shared only between $A_{e}\left(\tilde{z}, \tilde{c}_{1}\right)$ and $B_{e}\left(\tilde{z}^{\prime}, \tilde{c}_{1}^{\prime}\right)$. After this first communication, $s a_{a}-1$ transitions OUT wait $_{\text {are necessary for applying the }}$ final OUT $_{\text {eff }}$ :

$$
\begin{gathered}
A_{e}\left(\tilde{z}, \tilde{c}_{1}\right)\left|C_{e}\left(\tilde{z}^{\prime}, \tilde{c}_{1}^{\prime}\right) \stackrel{\dot{a}^{\prime}}{\longrightarrow}{ }_{e} \cdots A_{e}\left(\tilde{z}, \tilde{c}_{s a_{a}}\right)\right| C_{e}\left(\tilde{z}^{\prime}, \tilde{c}_{s a_{a}}^{\prime}\right) \\
\stackrel{a^{\prime \prime}}{\longrightarrow} B_{e}(\tilde{w}, \tilde{d}) \mid D\left(\tilde{w}^{\prime}, \tilde{d}^{\prime}\right)
\end{gathered}
$$

As $a^{\prime \prime}$ is an instantaneous transition and the rate of $a^{\prime}$ is the same as the rate of $a$, the duration of the chain follows an Erlang distribution of rate $r_{a}^{\prime}$ and stochasticity absorption $s a_{a}$.

Finally, from rules depicted in Figure 3, it can be deduced that $A_{e}(\tilde{z}, \tilde{c}) \stackrel{\pi}{\rightarrow}_{e} B_{e}(\tilde{w}, \tilde{d})$ implies $A(\tilde{z}) \stackrel{\pi}{\rightarrow}_{E} B(\tilde{w})$ - and there is a finite number of transitions between $A_{e}(\tilde{z}, \tilde{c})$ and $B_{e}(\tilde{w}, \tilde{d})$. From the previous points, it has been shown that the correct number of transition has to be done between the initial call of $A_{e}$ and its final transition to $B_{e}$. This ends the correction of the proposed construction of the stochasticity absorption factor.

\subsection{Toy Example}

We apply the results obtained in this section to a toy example: an infinite looping sequence of two processes (14).

$$
A_{0}::=\tau_{t} . A_{1} \quad A_{1}::=\tau_{t} . A_{0}
$$

Basically, starting from $A_{0}$, we expect to observe an infinite sequence of $A_{0}$ becoming $A_{1}$ becoming $A_{0}$, etc. Between each transition, the interval action $\tau_{t}$ is performed.

Figure 4 plots the presence of $A_{0}$ and $A_{1}$ during simulations of $\llbracket A_{0} \rrbracket_{e}$ under SPIM [8], [17] with an identical rate but different stochasticity absorption factors.

When no stochasticity absorption is applied, we observe a strong variance of the duration of the internal actions, as imposed by the exponential distribution. By increasing the stochasticity absorption factor, this variance reduces. Regular oscillations are observed with a high stochasticity absorption factor.

\section{FIRING INTERVALS}

The aim of this section is to point up a link between the couple use rate and stochasticity absorption factor of an action and the interval of time within it is fired 


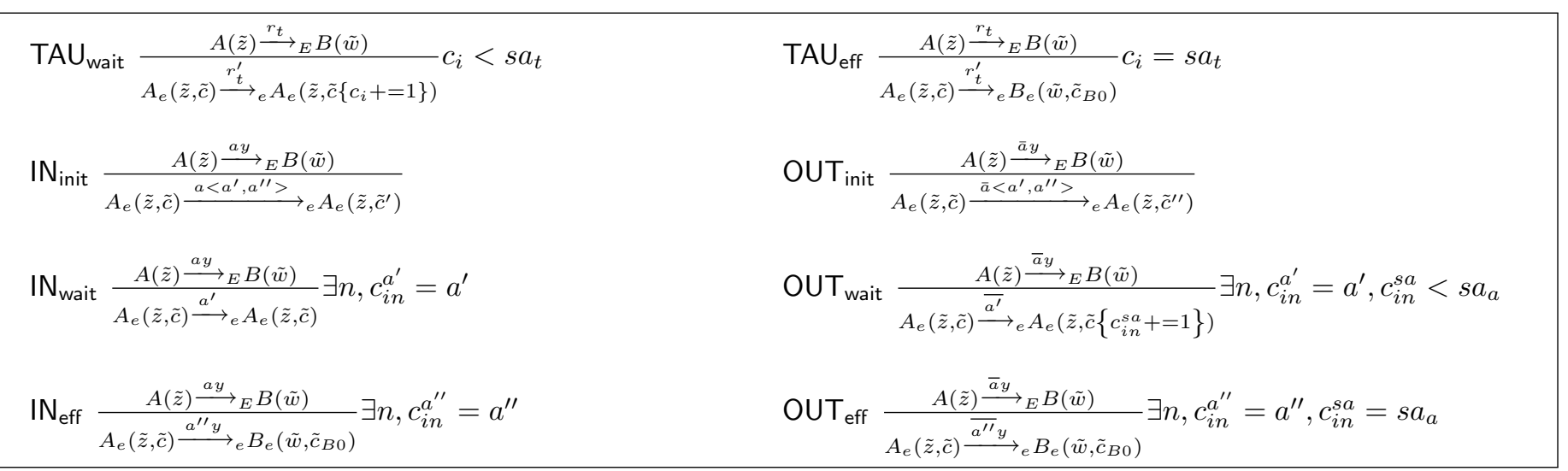

Fig. 3. Operational semantics derived from Definition 2. $i$ is the index of the action $\pi$ in the sum defined by the $S \pi_{E r}$ process $A . \tilde{c}^{\prime}$ and $\tilde{c}^{\prime \prime}$ are computed respectively as in (12) and (11). $\tilde{c}_{B 0}$ is computed as in (13).
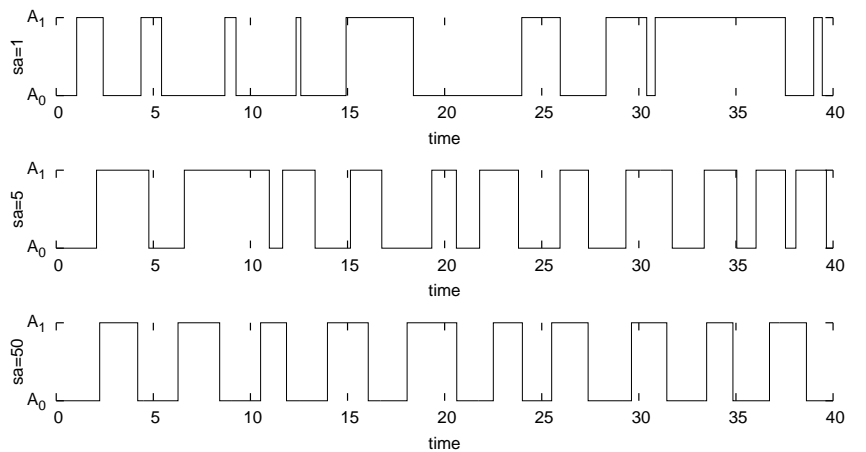

Fig. 4. Simulation of the $S \pi_{E r}$ process defined by (14) with rate $\frac{1}{2}$ and different absorption factors $s a$.

at a given confidence. That timed interval is refered as the firing interval (Definition 3). This section results in a set of statistical tools that may help the modelling and the checking of temporal and stochastic systems where durations of transitions follow an Erlang distribution.

Definition 3 (Firing Interval). Given $a$ use rate $r$ and a stochasticity absorption factor sa, the Firing Interval at confidence coefficient $1-\alpha$ is noted $F I_{\alpha}(r, s a)=[d ; D]$ where $F_{r, s a}(d)=\frac{\alpha}{2}$ and $F_{r, s a}(D)=1-\frac{\alpha}{2}$.

At the modelling time, and more especially when parametrising a model, it may be more natural to reason on firing intervals than on couples rate and stochasticity absorption factor. Here, we provide estimators and approximating functions to translate from and back a firing interval to rate and stochasticity absorption parameters.

These bring to the stochastic absorption factor different usages, depending on the knowledge and on the nature of the modelled system. On the one hand, the stochasticity absorption factor expresses a certainty on the precise action duration: the higher the confidence in the action duration is, the more the stochasticity absorption factor can be raisen. On the other hand, the stochasticity absorption supplies the ability to reproduce actions with intrinsic stochasticity where time bounds are known.

As supplementary modelling helpers, we points out there exists methods for inferring the shape [18] and the scale [19] parameters of a gamma distribution from a set of time measurement data. The conversion from gamma parameters to Erlang parameters is discussed below.

In the following of this section, the computation of the time interval from rate and stochasticity absorption parameters is first introduced. Finally, the computation of the rate and stochasticity absorption corresponding to a given time interval is tackled. To our knowledge, no prior study has been done in that way.

\subsection{From Parameters to Firing Interval}

Given the rate and stochasticity absorption parameters of an action, we are interested in computing the confidence interval for the time at which the action is fired. Let $1-$ $\alpha$ be the confidence coefficient for computing the firing interval. We search $d$ and $D$ such that $F_{r, s a}(d)=\frac{\alpha}{2}$ and $F_{r, s a}(D)=1-\frac{\alpha}{2}$, where $F_{r, s a}$ is the CDF of the Erlang distribution of use rate $r$ and stochasticity absorption factor $s a$ (2). The function which associates to $0 \leq x \leq 1$ the time $t$ such that $F_{r, s a}(t)=x$ is known as the quantile function, and is noted $F^{-1}$.

Because of its relation with the incomplete gamma function, the quantile function of the gamma distribution, hence of the Erlang distribution, has no easy analytical expression and can not be used directly [20]. However, efficient approximation algorithms for $F^{-1}$ exist [21], [22]. The widely used statistical tool $R$ [23] proposes an implementation of such an algorithm. As $R$ is distributed with a $C$ programming language library, one can easily access to this implementation from independent programs. To compute the quantiles for the Erlang distribution using $R$, the ggamma function can be used. Here is an instance of a $R$ session for computing the firing interval of an action of use rate $r$ and stochasticity absorption factor $s a$ :

$$
\begin{aligned}
d & \leftarrow \text { qgamma }(\alpha / 2, \text { shape }=s a, \text { rate }=r * s a) \\
D & \leftarrow \text { qgamma }(1-\alpha / 2, \text { shape }=s a, \text { rate }=r * s a)
\end{aligned}
$$




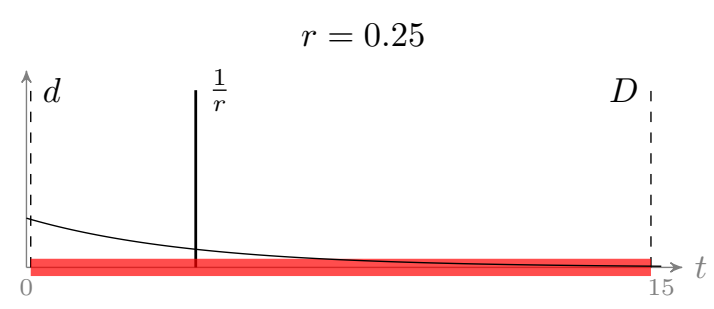

$s a=1 ; F I_{\alpha}(r, s a) \simeq[0.10127 ; 14.75552]$

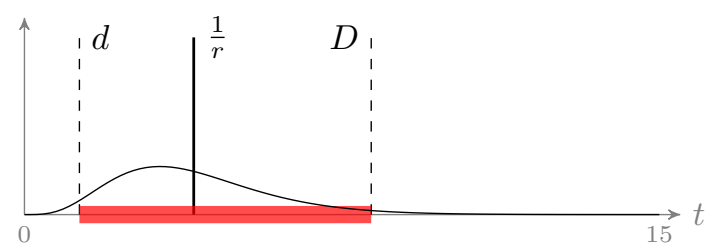

$s a=5 ; F I_{\alpha}(r, s a) \simeq[1.29879 ; 8.19327]$

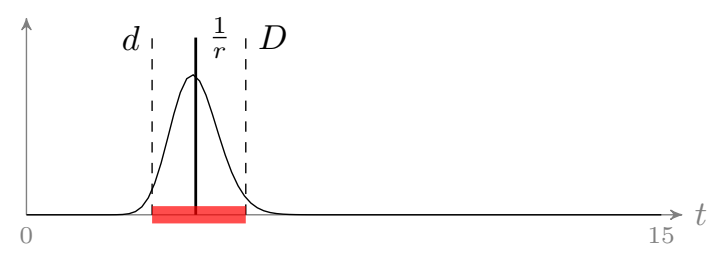

$s a=50 ; F I_{\alpha}(r, s a) \simeq[2.96888 ; 5.18245]$

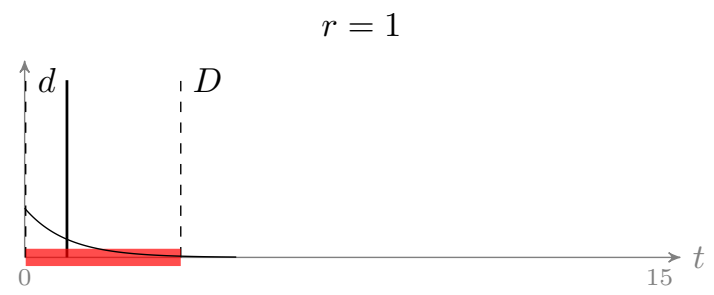

$s a=1 ; F I_{\alpha}(r, s a) \simeq[0.02532 ; 3.68888]$

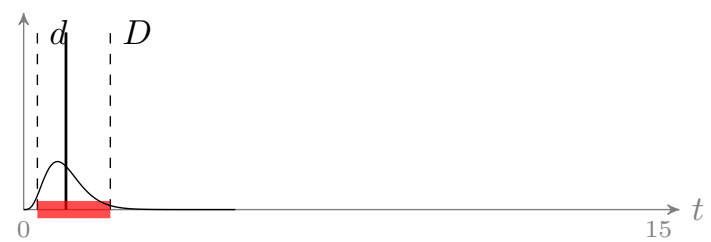

$s a=5 ; F I_{\alpha}(r, s a) \simeq[0.32470 ; 2.04832]$

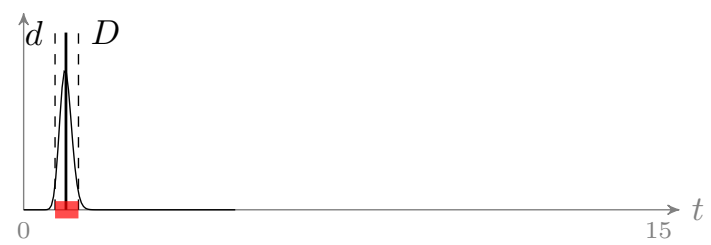

$s a=50 ; F I_{\alpha}(r, s a) \simeq[0.74222 ; 1.29561]$

Fig. 5. Evolution of the firing interval when the stochasticity absorption factor $s a$ increases. The thick vertical line is the average duration for each parameters. Confidence coefficient has been fixed to $95 \%(\alpha=0.05)$.

Figure 5 shows the influence of the use rate and the stochasticity absorption factor on the firing interval.

\subsection{From Firing Interval to Parameters}

Given a firing interval $[d ; D]$ at a confidence coefficient $1-\alpha$, we look for a rate $r$ and stochastic absorption factor $s a$ such that $F I_{\alpha}(r, s a)=[d, D]$. To achieve this goal, an estimator of $r$ and $s a$ (respectively $\hat{r}_{\alpha}$ and $\hat{s a} a_{\alpha}$ ) is built in function of the lower and upper bound of the firing interval $d$ and $D$.

In a first phase, the integer constraint on the stochasticity absorption factor is released to consider the gamma distribution of use rate $r \in \mathbb{R}_{+}^{*}$ and stochasticity absorption factor $s a \in \mathbb{R}_{+}^{*}$. As there is no analytical expression of the quantile function of the gamma distribution, expressing $r$ and $s a$ in function of the confidence interval can not be done analytically either. Therefore, to be able to build an estimator, firing intervals have been computed for a huge amount of use rates $r$ and stochasticity absorption factors. Estimators are then obtained by a regression on the generated data. For this report, $d$ and $D$ have been computed at a confidence coefficient of $95 \%$ for all rounded $\log s a$ between 0 and 4.5 (step of 0.1 ) and all $\log r$ between -8 and 2 (step of 0.1). Figure 6 shows the heat map for $r$ and $s a$ parameters in function of the bounds $d$ and $D$ of firing intervals for such a generation.

From this obtained 3-D plots, regressions fitting the data have been manually determined. Parameters of these regressions have then been abstracted as they may depend on the confidence coefficient value. The estimator obtained for the use rate, noted $\hat{r}_{\alpha}$, is given by (15), and the one for the stochasticity absorption factor, noted $\hat{s a} a_{\alpha}$ is given by (16).

$$
\begin{aligned}
\hat{r}_{\alpha} & =(w+x \exp (-y . d))(d+D)^{-1} \\
\hat{s} a_{\alpha} & =\exp \left(u\left(\frac{D}{d}\right)^{v}\right)
\end{aligned}
$$

where $u, v, w, x, y$ are parameters depending on the confidence coefficent $1-\alpha$.

Finally, $R$ has been used to valuate the parameters of these estimators from a set of generated data points. Table 1 sums up the estimators found for $r$ ans $s a$ at different confidence coefficients. The lack of usable analytical expressions around the gamma distribution makes it difficult to evaluate. Figure 7 shows an attempt to evaluate the quality of these estimators by comparing, for generated data, the expected value and the estimated value.

It is worth to notice that the estimators are bijective functions, i.e. to each firing interval corresponds one and only one use rate and stochasticity absorption factor. The resting point is the integer constraints on the stochasticity absorption factor. Because of the bijectivity of the estimators, if the estimated stochasticity absorption is not an integer, there is no Erlang distribution fitting with the given firing interval. From this observation, the search for approximating Erlang distribution parameters 
is at the confidence of the modeller. However, one can notice that rounding the estimated stochasticity absorption factor to the upward (resp. downward) integer results in a firing interval included by (resp. including the) originally given firing interval. In that way, one can easily estimate an over or under approximation of the couple of parameters matching an arbitrary firing interval.

\subsection{Sequence of Actions}

To conclude this section, we briefly discuss about the distribution of a sequence of actions and its relation with firing intervals.

Given $k$ actions at respectively rates $r_{1}, \ldots, r_{k}$ and stochasticity absorption factors $s a_{1}, \ldots, s a_{k}$, and considering they are fired successively, what is the firing interval of the sum of the actions?

It can be easily checked that the firing interval $[d ; D]$ of a sequence of Erlang distributed actions is not the sum of the firing intervals of the individual actions i.e. $d \neq d_{1}+\cdots+d_{k}$ where $d_{i}$ is the lower bound of the firing interval of the $i^{t h}$ action ; $D$ is computed similarly. However, the sum of Erlang distributed random variables with different parameters has been studied in [24], [25] and [26] gives an easily computable expression of the CDF for such a distribution. In that way, the firing interval of the sum of Erlang distributed random variables can be computed using standard approximation techniques of the quantile function (like bisections). The dual operation consisting of inferring the parameters of the Erlang sum from the firing interval raises several difficulties, such as the lost of bijectivity. We consider such an issue as out of the scope of this report.

\section{MOdel CHECKING USING PRISM}

The probabilist model checker PRISM [9] offers efficient model checking for CTMCs. In PRISM, actions are defined within PRISM modules. Each module has a finite set of local variables. The union of the local variables of all modules gives the global state of the model, denoted by $V$.

$$
[\text { act }] \text { guard } \rightarrow r:\left(x_{1}^{\prime}=u_{1}\right) \& \ldots \&\left(x_{k}^{\prime}=u_{k}\right)
$$

where act is an optional action label, guard a predicate over $V, x_{i}$ is a local variable and $u_{i}$ a function over $V$. $r \in \mathbb{R}_{+}^{*}$ is the use rate of the action and is assumed to 1 when omitted. To be applicable, a labelled action has to be synchronised with an action of the same label in another module. The rate of such a synchronised action is the product of the rate of both actions.

An efficient translation of the classical exponentially distributed stochastic $\pi$-calculus $\left(S \pi_{e}\right)$ into PRISM has been proposed by Norman et al. [12]. Their translation requires the overall process structure to be rearrangeable to the form $P=\nu x_{1} \ldots \nu x_{k}\left(P_{1}|\ldots| P_{n}\right)$ where each $P_{i}$ contains no $\nu$ operator nor recursive use of the $\mid$ operator, especially to ensure a finite number of states. In that way, given a $S \pi_{E r}$ process $P$ respecting these constraints, the constructed $S \pi_{e}$ process $\llbracket P \rrbracket_{e}$ does not respect this limitation. Indeed, our proposed construction of the output action into $S \pi_{e}$, presented in (11), involves a recursive generation of fresh names $a^{\prime}, a^{\prime \prime}$. A solution is then to directly translate the $S \pi_{E r}$ process to PRISM.

In this section, the translation of $S \pi_{e}$ process to PRISM proposed by Normal et al. is adapted to the translation of $S \pi_{E r}$ process respecting previously cited structure. Therefore, this allows an efficient model checking of $S \pi_{E r}$ processes, which is a new result.

To end, the overall approach of this report is illustrated by a toy example.

\subsection{Construction of the Stochasticity Absorption}

Let $P$ be a stochastic $\pi$-calculus expression of the form $P=\nu x_{1} \ldots \nu x_{k}\left(P_{1}|\ldots| P_{n}\right)$ where each $P_{i}$ contains neither the $\nu$ or $\mid$ operator. In that way, each process $P_{i}$ can be described by a transition graph where nodes are race conditions annotated by $Q_{i}, R_{i}, \ldots$ [12]. Hence, each $Q_{i}, R_{i}, \ldots$ stands for a state of the process $P_{i}$. Using the translation detailed in [12], the PRISM model corresponding to $P$ can be computed. It results in $n$ PRISM modules, one per $P_{i}$, each having a variable $s_{i}$ representing the current state of the process $P_{i}$. Variables representing names to be sent or to be received are also attached to modules. Obtained actions are of three different main forms:

$$
\begin{aligned}
{[]\left(s_{i}=Q_{i}\right) \& M } & \rightarrow r_{t}:\left(s_{i}^{\prime}=R_{i}\right) \\
{\left[a_{-} P_{i \_} P_{j \_} y\right] } & \left(s_{i}=Q_{i}\right) \& M \rightarrow r_{a}:\left(s_{i}^{\prime}=R_{i}\right) \\
{\left[a_{-} P_{j \_} P_{i \_} y\right] } & \left(s_{i}=Q_{i}\right) \& M \rightarrow\left(s_{i}^{\prime}=R_{i}\right) \&\left(z^{\prime}=y\right)
\end{aligned}
$$

Each of these forms represents the process $P_{i}$ at state $Q_{i}$ applying a certain action under the condition $M$ and changing to state $R_{i}$. These actions are respectively the internal action (17), the output (either bound or free) of $y$ on a channel $a$ to $P_{j}$ (18) and the input of $y$ as $z$ on a channel $a$ from $P_{j}$ (19). Depending on the boundness of the sent name $y$, supplementary conditions are added to $M$. In the following of this section, we assume that any identical action label inside a same module have disjoint guards, i.e. both are never part of a same race condition.

As for the construction of the stochasticity absorption factor in $S \pi_{e}$, a counter is attached to each PRISM action. For identifying internal actions, a label is attached to them: $P_{i_{-}} t$ is the label of the internal action $\tau_{t}$ of $P_{i}$. The set of labels of internal and output actions of $P_{i}$ is denoted by $\mathcal{L}_{P_{i}} \diamond=\left\{a \_P_{i_{-}} P_{j} y, \ldots, P_{i_{-}} t, \ldots\right\}$, and the set of labels of input actions of $P_{i}$ is denoted by $\mathcal{L}_{\diamond P_{i}}=\left\{a \_P_{j \_} P_{i \_} y, \ldots\right\}$. Basically, there is one counter for each action having a label in $l \in \mathcal{L}_{P_{i} \diamond}$. This counter is defined as a local variable $c_{-} l$ of the PRISM module for $P_{i}$. Each time an output or an internal action is performed, the corresponding counter is incremented by one. The update of the action is proceed when this 


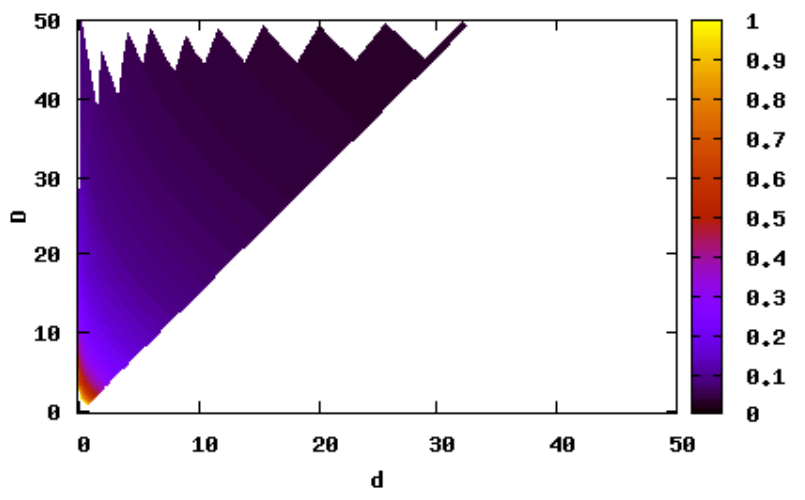

$r_{0.05}$

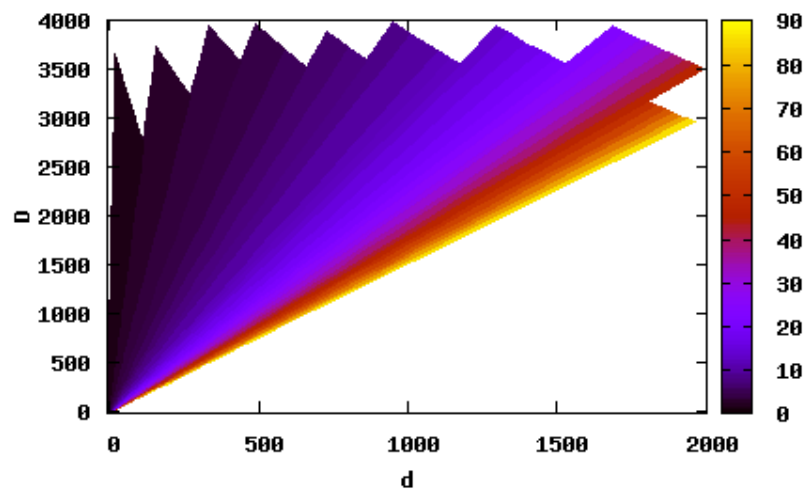

$s a_{0.05}$

Fig. 6. Heat map of the use rate (left) and stochasticity absorption factor (right) in function of the bounds $d$ and $D$ of the Firing Interval $F I_{0.05}=[d, D]$ for some generated data.

TABLE 1

Obtained estimators of parameters $r$ and $s a$ for the Firing Interval $[d ; D]$ at different confidence coefficients.

\begin{tabular}{|c|c|c|c|}
\hline & $1-\alpha=0.90$ & $1-\alpha=0.95$ & $1-\alpha=0.99$ \\
\hline$\hat{r}_{\alpha}$ & $(2.13+2.89 \exp (-44.46 d))(d+D)^{-1}$ & $(2.06+1.93 \exp (-36.49 d))(d+D)^{-1}$ & $(2.03+1.39 \exp (-33.33 d))(d+D)^{-1}$ \\
\hline$\hat{s} a_{\alpha}$ & $\exp \left(6.39\left(\frac{d}{D}\right)^{-0.66}\right)$ & $\exp \left(6.41\left(\frac{d}{D}\right)^{-0.87}\right)$ & $\exp \left(6.41\left(\frac{d}{D}\right)^{-1.04}\right)$ \\
\hline
\end{tabular}

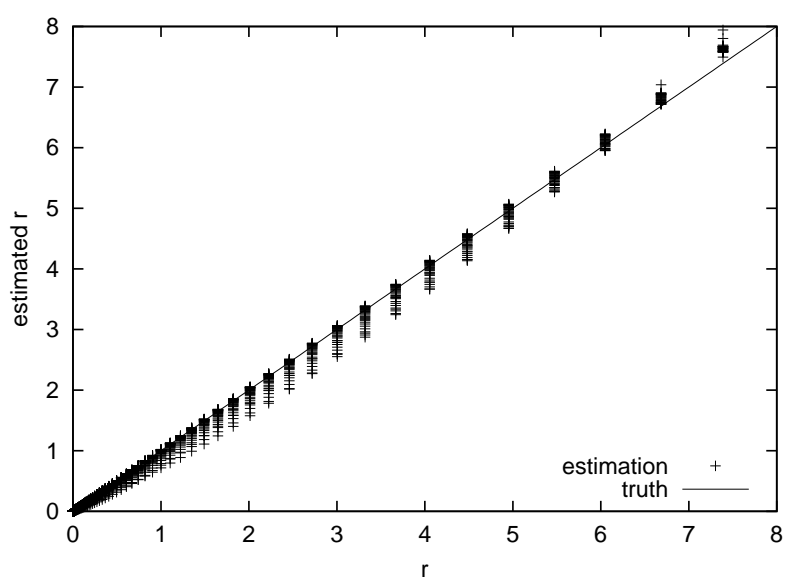

evaluation of $\hat{r}_{0.05}$

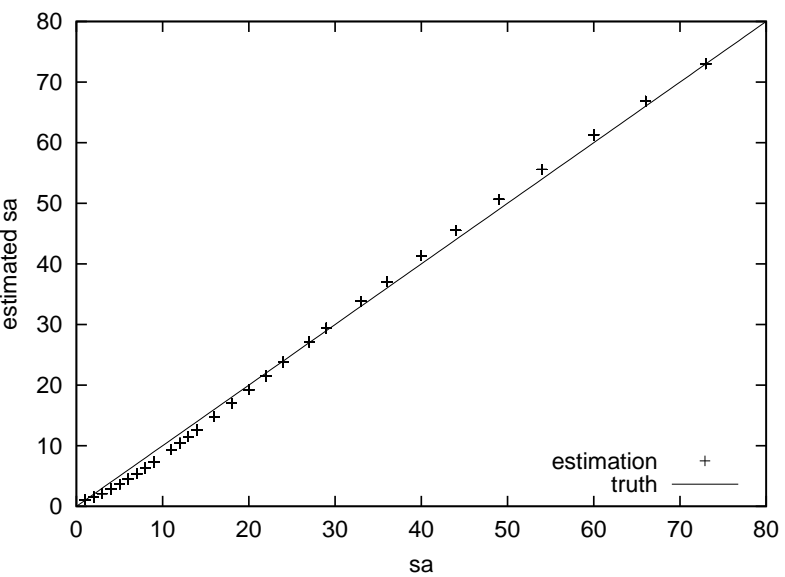

evaluation of $\hat{s a} a_{0.05}$

Fig. 7. Evaluation of obtained estimators for use rate $r$ and stochasticity absorption factor $s a$ with confidence coefficient of $95 \%$. Points are the estimated parameter value. A perfect estimator would put all points on the truth line.

counter reaches the expected stochasticity absorption factor.

To ensure a correct count of absorption, all counters related $P_{i}$ have to be reset when $P_{i}$ changes its state. This raises a concern concerning the reset of counters not owned by $P_{i}$, as this is the case for all actions labelled by $\mathcal{L}_{\diamond P_{i}}$. To cope with such an issue, a boolean variable $d_{-} l$ is defined in the module $P_{i}$ for each $l=a_{-} P_{j} P_{i} \_y \in$ $\mathcal{L}_{\diamond P_{i}}$, and is set to true when the reset of $c_{-} l$ is required.

The following subsection precisely describes transformations to apply to each of the PRISM modules $P_{i}$ to add support for the stochasticity absorption factor.

\section{Additional local variables}

For each label $l \in \mathcal{L}_{P_{i} \diamond}$, the variable $c_{-} l$ stands for the counter of the stochasticity absorption.

$$
c \_l:\left[1 . . s a \_l\right] \text { init } 1 \text {; }
$$

For each label $l \in \mathcal{L}_{\diamond P_{i}}$, the boolean variable $d_{-} l$ is true if $P_{i}$ has changed its state while the absorption of the action played by $l$ has started. In other terms, $d \_l$ is 
true if the associated counter $c_{-} l$ has to be reset.

$$
\text { d_l : bool init false; }
$$

Hereafter, the PRISM update for the reset of all stochasticity absorption counter is denoted by $R_{P_{i} \diamond}(20)$. The update of $d_{-} l$ variables, $l \in \mathcal{L}_{\diamond P_{i}}$, is denoted by $S_{\diamond P_{i}}$ (21). Basically, $d_{-} l$ is set to true if and only if the associated counter $c_{-} l$ is different from its default value.

$$
\begin{aligned}
& R_{P_{i} \diamond} \stackrel{\text { def }}{=} \underset{l \in \mathcal{L}_{P_{i} \diamond}}{\mathcal{L}_{\Delta}}\left(c_{-} l^{\prime}=1\right) \\
& S_{\diamond P_{i}} \stackrel{\text { def }}{=} \underset{l \in \mathcal{L}_{\diamond P_{i}}}{\&_{-}}\left(d l^{\prime}=c_{-} l>1\right)
\end{aligned}
$$

with $\underset{i \in\{1, \ldots, k\}}{\&} u_{i}=u_{1} \& \cdots \& u_{k}$.

\section{Internal action}

Let $l=P_{i_{-}} t$ be the label of an action resulting from the translation of an internal action $\tau_{t}$. The use rate and stochasticity absorption factor of this internal action are respectively $r_{t}$ and $s a_{t}$. The PRISM action respects the following form:

$$
\text { [] } G \rightarrow r_{t}: U
$$

where $G$ stands for the guard of the action and $U$ for the updates to perform. The stochasticity absorption of the action is achieved by replacing the previous statement by the actions below:

$$
\begin{aligned}
& \text { [] } G \&\left(c_{-} l<s a_{t}\right) \rightarrow r_{t} * s a_{t}:\left(c_{-} l^{\prime}=c_{-} l+1\right) ; \\
& \text { [] } G \&\left(c_{-} l=s a_{t}\right) \rightarrow r_{t} * s a_{t}: U \& R_{P_{i}} \diamond \& S_{\diamond P_{i}} ;
\end{aligned}
$$

\section{Channel output}

Let $l=a_{-} P_{i \_} P_{j} y$ be the label an action resulting from the translation of an output of name $y$ on channel $a$. The use rate and stochasticity absorption factor of this channel are respectively $r_{a}$ and $s a_{a}$. The PRISM action respects the following form:

$$
[l] G \rightarrow r_{a}: U
$$

where $G$ stands for the guard of the action and $U$ for the updates to perform. The stochasticity absorption of the action is achieved by replacing the previous statement by the actions below.

$$
\begin{aligned}
& {\left[l \_w a i t\right] G \& d \_l \rightarrow r_{a} * s a_{a}:\left(c \_l^{\prime}=2\right) ;} \\
& {\left[l \_w a i t\right] G \& ! d \_l \&\left(c_{-} l<s a_{a}\right) \rightarrow r_{a} * s a_{a}:} \\
& \left(c_{-} l^{\prime}=c_{-} l+1\right) ; \\
& {[l] G \& ! d \_l \&\left(c_{-} l=s a_{a}\right) \rightarrow r_{a} * s a_{a}:} \\
& U \& R_{P_{i} \diamond} \& S_{\diamond P_{i}} ;
\end{aligned}
$$

\section{Channel input}

Let $l=a \_P_{j} P_{i} \_y$ be the label of an action resulting from the translation of an input of name $y$ on channel $a$. The PRISM action respects the following form:

$$
[l] G \rightarrow U
$$

where $G$ stands for the guard of the action and $U$ for the updates to perform. The stochasticity absorption of the action is achieved by replacing the previous statement by the actions below:

$$
\begin{aligned}
& {\left[l \_ \text {wait }\right] G \rightarrow\left(d_{-} l^{\prime}=\text { false }\right)} \\
& {[l] G \rightarrow U \& R_{P_{i} \diamond} \& S_{\diamond P_{i}} ;}
\end{aligned}
$$

\subsection{Toy Example}

As an application of the use of the PRISM model checker for analysing $S \pi_{E r}$ processes, and as an illustration of the overall method presented by this report, we propose the study of an example process $P$ defined in (22).

$$
\begin{array}{cl}
A_{1}::=a \cdot A_{0}+\bar{b} \cdot A_{1} \quad \begin{array}{r}
B_{1}::=b \cdot B_{0}+\bar{a} \cdot B_{1} \\
A_{0}::=\perp \quad \\
B_{0}::=\perp
\end{array} \\
P::=\nu a \nu b \nu \perp\left(A_{1} \mid B_{1}\right)
\end{array}
$$

Intuitively, two stories can happen: either $A_{1}$ outputs first on $b$ then $B_{1}$ becomes $B_{0}$ and the system ends in deadlock ; or $B_{1}$ outputs first on $a$ then $A_{1}$ becomes $A_{0}$ and the system ends in deadlock.

For this toy example, we search for reducing to near zero the probability that $A_{1}$ becomes $A_{0}$, i.e. the probability $p$ that $B_{1}$ outputs on $a$. As supplementary constraints, the average duration for using channels $a$ and $b$ is fixed to respectively 4 and 1 time units (i.e. $r_{a}=0.25$ and $\left.r_{b}=1\right)$. For instance, these constraints may have been imposed by observations of the real system modelled by $P$. The property to verify is expressed in PRISM as $\mathbf{P}=$ ? $\left[\mathbf{F} \quad\left(\mathrm{s} \_\mathrm{a}=0\right)\right]$ which means the probability that $A_{0}$ is reached.

We first study this model as a $S \pi_{e}$ process. Listing 1 shows the result of the translation of $P$ into PRISM using the method of Norman et al. Verifying the previously given property with PRISM results in a probability for reaching $A_{0}$ of 0.2 .

Let us consider $P$ as a $S \pi_{E r}$ process. For the sake of simplicity, we will consider that both $a$ and $b$ have the same stochasticity absorption factor.

In terms of firing interval, we look for a stochasticity absorption factor with which the firing interval of $b$ is entirely before the firing interval of $a$. By computing the firing interval for both of these actions at different absorptions factors - as done in Figure 8 - one can observe the probability of firing $a$ before $b$ will be seriously decreased with a stochasticity absorption factor of 5 . By using a stochasticity absorption factor of 50 , we expect the probability of firing $a$ first to be near zero.

To confirm these results, we now turn to the model checking of the $S \pi_{E r}$ process $P$ using PRISM. Listing 2 shows the translation of $P$ into PRISM using the construction presented above. With a stochasticity absorption of 5 , the probability that $A_{1}$ reaches $A_{0}$ is divided by 100 (almost 0.02) comparing without stochasticity absorption. As expected, increasing this stochasticity absorption factor to 50 drops down this probability to approximately $10^{-11}$. 


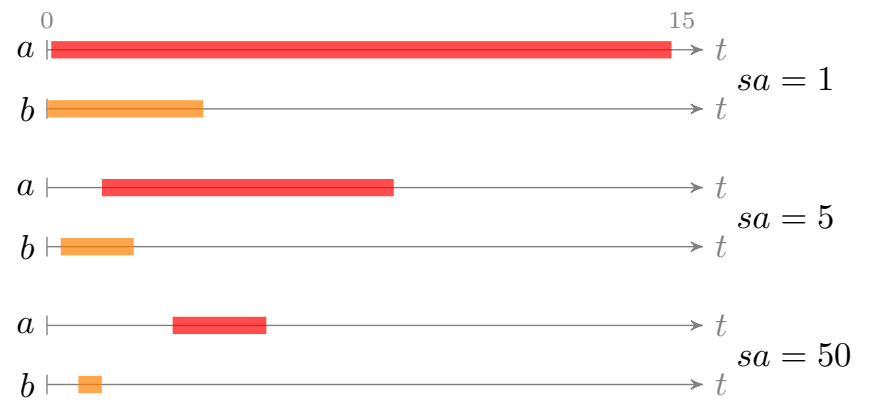

Fig. 8. Firing intervals at confidence coefficient $95 \%$ for $a$ and $b$ (22) with different stochasticity absorption factors but constant use rates $r_{a}=0.25$ and $r_{b}=1$.

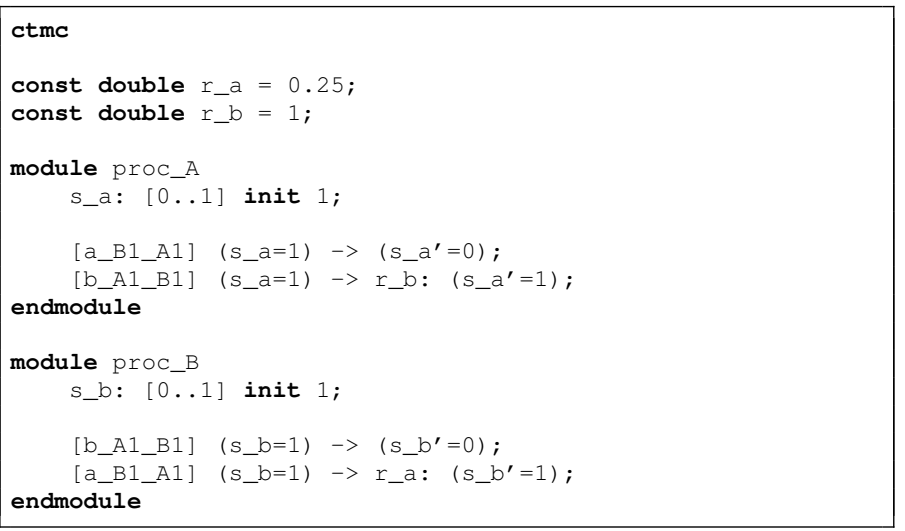

Listing 1. Translation of the example stochastic $\pi$ calculus model (22) as a $S \pi_{e}$ process into PRISM.

\section{Conclusion}

In this report, we presented and proved a technique for tuning temporal features within the stochastic $\pi$ calculus. This tuning is done through a stochasticity absorption factor which reduces the variance around the average duration of transitions. This absorption is achieved by replacing the exponential distribution for firing actions by the Erlang distribution.

We proved a translation of the Erlang distributed stochastic $\pi$-calculus into the exponential distributed one. In this way, such tuned models can still be efficiently simulated with standard algorithms. The assignment of the temporal and stochastic parameters has been discussed and we detailled the link with the classical timed interval for firing transitions. We showed that by using estimators it is possible to parameterise stochastic $\pi$-calculus models by specifying either the parameters of the Erlang distribution or the firing interval at a given confidence level. Finally, we added support for this stochasticity absorption factor within the translation of the stochastic $\pi$-calclus to PRISM, allowing efficient model-checking of these models.

As further works, we are interested in improving the efficienty of the model-checking for Erlang distributed stochastic $\pi$-calculus by the study of the sum of Erlang random variables with different parameters.

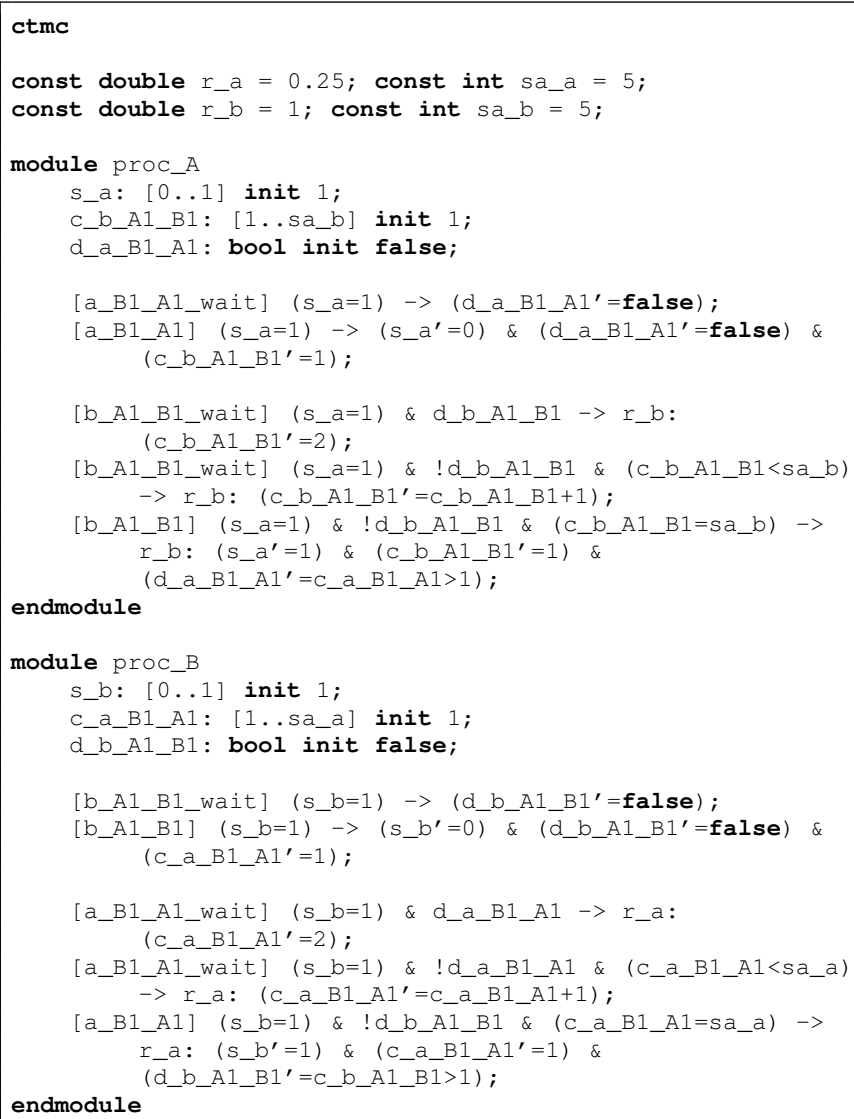

Listing 2. Translation of the example stochastic $\pi$ calculus model (22) as a $S \pi_{E r}$ process into PRISM.

\section{APPENDIX \\ AN ALTERNATIVE CONSTRUCTION FOR THE STOCHASTICITY ABSORPTION FACTOR WITHIN THE STOCHASTIC $\pi$-CALCULUS}

In this appendix, we provide an alternative to that of Definition 2 for the construction of the stochasticity absorption factor within the stochastic $\pi$-calculus. The main motivation for this new construction is to prevent the use of an indexing set for the race condition which depend on parameters of the process. That is the case for the proposed construction of the channel output (11) and input (11). The construction hereafter can then be fully implemented in SPIM, for instance.

Basically, instead of having a race condition growing after each communication instantiation, a process handling the stochasticity absorption for each new communication is composed in parallel. To identify the process $P_{i}$ winning the race condition $\sum_{i \in I} P_{i}$, a name $c_{i}$ is bound to each component of the race condition. When the process handling a communication has completed its absorption, it outputs on this channel $c_{i}$. In that way, the translation of the call for the race condition (13) is replaced by:

$$
\llbracket A(\tilde{z}) \rrbracket_{e}=A(\tilde{z}, \tilde{c})
$$


with $\forall i \in I, c_{i}= \begin{cases}1 & \begin{array}{l}\text { if the first action of } P_{i} \\ \text { is an internal action, }\end{array} \\ \nu c_{i} & \text { else, with } r_{c_{i}}=\infty\end{cases}$

Channel output: The process defined below takes as parameters the stochasticity absorption factor to reach $s a$, the counter of firing $c$, channels $a^{\prime}, a^{\prime \prime}$ as defined in (11), and the channel $r$ for outputting when the absorption is completed.

$$
\begin{gathered}
A_{\text {send }}\left(s a, c, a^{\prime}, a^{\prime \prime}, r\right)::= \\
{[c<s a] \overline{a^{\prime}} \cdot A_{\text {send }}\left(s a, c+1, a^{\prime}, a^{\prime \prime}, r\right)} \\
+[c=s a] \bar{r} a^{\prime \prime}
\end{gathered}
$$

(11) is then replaced by the following:

$$
\begin{aligned}
& \llbracket \bar{a} y \cdot P \rrbracket_{e}= \\
& \nu a^{\prime} \nu a^{\prime \prime} \bar{a}<a^{\prime}, a^{\prime \prime}>.\left(A(\tilde{z}, \tilde{c}) \mid A_{\text {send }}\left(s a_{a}, 1, a^{\prime}, a^{\prime \prime}, c_{i}\right)\right) \\
& \quad+c_{i} a^{\prime \prime} \cdot \overline{a^{\prime \prime}} y \cdot \llbracket P \rrbracket_{e}
\end{aligned}
$$

with $r_{a^{\prime}}^{\prime}=r_{a^{\prime}}^{\prime} r_{a^{\prime \prime}}^{\prime}=\infty$ and $\left\{a^{\prime}, a^{\prime \prime}\right\} \cap(f n(A) \cup f n(P))$.

Channel input: Similarly, the process defined below inputs either on the channel $a^{\prime}$ (absorption in progress) on the channel $a^{\prime \prime}$ (communication done), and outputs on $r$ the data received by $a^{\prime \prime}$ to the parent race condition.

$$
\begin{aligned}
A_{\text {recv }}\left(a^{\prime}, a^{\prime \prime}, r\right)::= & a^{\prime} \cdot A_{r e c v}\left(a^{\prime}, a^{\prime \prime}, r\right) \\
& +a^{\prime \prime} y \cdot \bar{r} y
\end{aligned}
$$

Finally, (12) is replaced as follows:

$$
\begin{aligned}
\llbracket a y . P \rrbracket_{e}= & a<a^{\prime}, a^{\prime \prime}>\cdot\left(A(\tilde{z}, \tilde{c}) \mid A_{r e c v}\left(a^{\prime}, a^{\prime \prime}, c_{i}\right)\right) \\
& +c_{i} y \cdot \llbracket P \rrbracket_{e}
\end{aligned}
$$

with $\left\{a^{\prime}, a^{\prime \prime}\right\} \cap(f n(A) \cup f n(P))=\emptyset$.

\section{REFERENCES}

[1] R. Milner, Communicating and mobile systems: the $\pi$-calculus. New York, NY, USA: Cambridge University Press, 1999.

[2] C. Priami, "Stochastic pi-Calculus," The Computer Journal, vol. 38, no. 7 , pp. $578-589,1995$.

[3] C. Priami, A. Regev, E. Shapiro, and W. Silverman, "Application of a stochastic name-passing calculus to representation and simulation of molecular processes," Inf. Process. Lett., vol. 80, no. 1, pp. 25-31, 2001.

[4] C. Kuttler and J. Niehren, "Gene regulation in the pi calculus: Simulating cooperativity at the lambda switch," Transactions on Computational Systems Biology, vol. 4230, no. VII, pp. 24-55, Nov. 2006.

[5] R. Blossey, L. Cardelli, and A. Phillips, "Compositionality, stochasticity and cooperativity in dynamic models of gene regulation," HFSP Journal, vol. 2, no. 1, pp. 17-28, Feb 2008.

[6] L. Cardelli, E. Caron, P. Gardner, O. Kahramanogullari, and A. Phillips, "A process model of actin polymerisation," Electronic Notes in Theoretical Computer Science, vol. 229, no. 1, pp. 127-144, January 2009

[7] D. T. Gillespie, "Exact stochastic simulation of coupled chemical reactions," The Journal of Physical Chemistry, vol. 81, no. 25, pp. 2340-2361, 1977.

[8] A. Phillips and L. Cardelli, "Efficient, correct simulation of biological processes in the stochastic pi-calculus," in Computational Methods in Systems Biology, ser. LNCS, vol. 4695. Springer, Sep 2007, pp. 184-199.
[9] A. Hinton, M. Kwiatkowska, G. Norman, and D. Parker, "PRISM: A tool for automatic verification of probabilistic systems," in Proc. 12th International Conference on Tools and Algorithms for the Construction and Analysis of Systems (TACAS'06), ser. LNCS, H. Hermanns and J. Palsberg, Eds., vol. 3920. Springer, 2006, pp. $441-444$.

[10] R. Alur and D. Dill, Lecture Notes in Computer Science, 1992, ch. The theory of timed automata, pp. 45-73.

[11] C. Priami, "Stochastic $\pi$-calculus with general distributions," in Proc. of the 4th Workshop on Process Algebras and Performance Modelling, CLUT, 1996, pp. 41-57.

[12] G. Norman, C. Palamidessi, D. Parker, and P. Wu, "Model checking probabilistic and stochastic extensions of the $\pi$-calculus," IEEE Transactions on Software Engineering, vol. 35, no. 2, pp. 209-223, 2009.

[13] M. Kwiatkowska, G. Norman, D. Parker, and J. Sproston, "Performance analysis of probabilistic timed automata using digital clocks," Formal Methods in System Design, vol. 29, pp. 33-78, 2006.

[14] A. Aziz, K. Sanwal, V. Singhal, and R. Brayton, "Model-checking continuous-time markov chains," ACM Trans. Comput. Logic, vol. 1 , no. 1, pp. $162-170,2000$

[15] C. Baier and H. Hermanns, "Model-checking algorithms for continuous-time markov chains," IEEE Trans. Softw. Eng., vol. 29, no. 6, pp. 524-541, 2003, senior Member-Haverkort, Boudewijn and Member-Katoen, Joost- Pieter.

[16] S. Donatelli, S. Haddad, and J. Sproston, "Model checking timed and stochastic properties with CSL ${ }^{T A}$," IEEE Transactions on Software Engineering, vol. 35, no. 2, pp. 224-240, 2009.

[17] A. Phillips, SPiM, http://research.microsoft.com/ aphillip/spim.

[18] A. Zaigraev and A. Podraza-Karakulska, "On estimation of the shape parameter of the gamma distribution," Statistics \& Probability Letters, vol. 78, no. 3, pp. 286 - 295, 2008.

[19] J. Mi and A. Naranjo, "Inferences about the scale parameter of the gamma distribution based on data mixed from censoring and grouping," Statistics \& Probability Letters, vol. 62, no. 3, pp. 229 243, 2003.

[20] D. J. Wilkinson, Stochastic Modelling for Systems Biology (Mathematical and Computational Biology). Chapman \& Hall/CRC, April 2006.

[21] D. Best and D. Roberts, "Algorithm as 91: The percentage points of the chi-squared distribution," Applied Statistics, vol. 24, no. 3, pp. 385-390, 1975.

[22] A. R. DiDonato and A. H. Morris, Jr., "Computation of the incomplete gamma function ratios and their inverse," ACM Trans. Math. Softw., vol. 12, no. 4, pp. 377-393, 1986

[23] R Development Core Team, R: A Language and Environment for Statistical Computing, R Foundation for Statistical Computing, Vienna, Austria, 2009, ISBN 3-900051-07-0. [Online]. Available: http://www.R-project.org

[24] S. Amari and R. Misra, "Closed-form expressions for distribution of sum of exponential random variables," Reliability, IEEE Transactions on, vol. 46, no. 4, pp. 519-522, Dec 1997.

[25] S. Nadarajah, "A review of results on sums of random variables," Acta Applicandae Mathematicae: An International Survey Journal on Applying Mathematics and Mathematical Applications, vol. 103, no. 2, pp. 131-140, Sep 2008.

[26] S. Favaro and S. Walker, "On the distribution of sums of independent exponential random variables via wilks' integral representation," Acta Applicandae Mathematicae: An International Survey Journal on Applying Mathematics and Mathematical Applications, 2008. 